\title{
A New Method for Performing Continuous Manometry during Pleural Effusion Drainage
}

\author{
Matthew Salamonsen ${ }^{a}$ Robert Ware ${ }^{b}$ David Fielding ${ }^{a}$

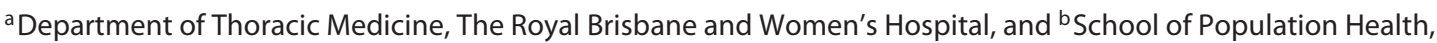 \\ The University of Queensland, Brisbane, Qld., Australia
}

\section{Key Words}

Pleural pressure · Manometry · Pleural effusion

management

\begin{abstract}
Background: Pleural manometry can predict the presence of trapped lung and guide large-volume thoracentesis. The current technique for pleural manometry transduces pressure from the needle or intercostal catheter, necessitating intermittent cessation of fluid drainage at the time of pressure recordings. Objectives: To develop and validate a technique for performing continuous pleural manometry, where pressure is transduced from an epidural catheter that is passed through the drainage tube to sit within the pleural space. Methods: Pleural manometry was performed on 10 patients undergoing thoracentesis of at least $500 \mathrm{ml}$, using the traditional intermittent and new continuous technique simultaneously, and pleural pressures were recorded after each drainage of $100 \mathrm{ml}$. The pleural elastance (PEL) curves and their $95 \%$ confidence intervals (Cls), derived using measurements from each technique, were compared using the analysis of covariance and Student's paired t test, respectively. Results: There was no significant difference in PEL calculated using each method ( $p>0.1$ ); however, there was a trend towards the $\mathrm{Cl}$ for the PEL derived from the continuous method being narrower ( $p=0.08$ ). Fully automated measurement of drainage volume and pleural pressure, with re-
\end{abstract}

al-time calculation and display of PEL, was achieved by connecting the system to a urodynamics machine. Conclusions: Pleural manometry can be transduced from an epidural catheter passed through the drainage tube into the pleural space, which gives continuous recording of the pleural pressure throughout the procedure. This allows for automated calculation and display of the pleural pressure and PEL in real time, if the system is connected to a computer with appropriate software.

(c) 2014 S. Karger AG, Basel

\section{Introduction}

Pleural manometry during drainage of a pleural effusion can predict the presence of unexpandable lung [1-3] and guide safe large-volume thoracentesis [4]. However, most pulmonologists are not familiar with the technique for pleural manometry and so it is not commonly performed $[5,6]$.

The traditional method for measuring pleural pressure during thoracentesis is by connection of a manometer to the thoracentesis needle or intercostal catheter [7, 8]. With this method, drainage must be temporarily ceased at the time of pressure recordings because any flow of fluid past the pressure manometer will invalidate the measurements. We have found this intermittent technique (i.e. the intermittent method) to be somewhat cum-

\section{KARGER}

E-Mail karger@karger.com

www.karger.com/res
C 2014 S. Karger AG, Basel

0025-7931/14/0881-0061\$39.50/0
Matthew Salamonsen

Department of Thoracic Medicine

The Royal Brisbane and Women's Hospital

Butterfield St. Herston, Qld. 4029 (Australia)

E-Mail mattsalamonsen@gmail.com 
Fig. 1. Procedure setup for pleural manometry using the intermittent and continuous methods simultaneously.

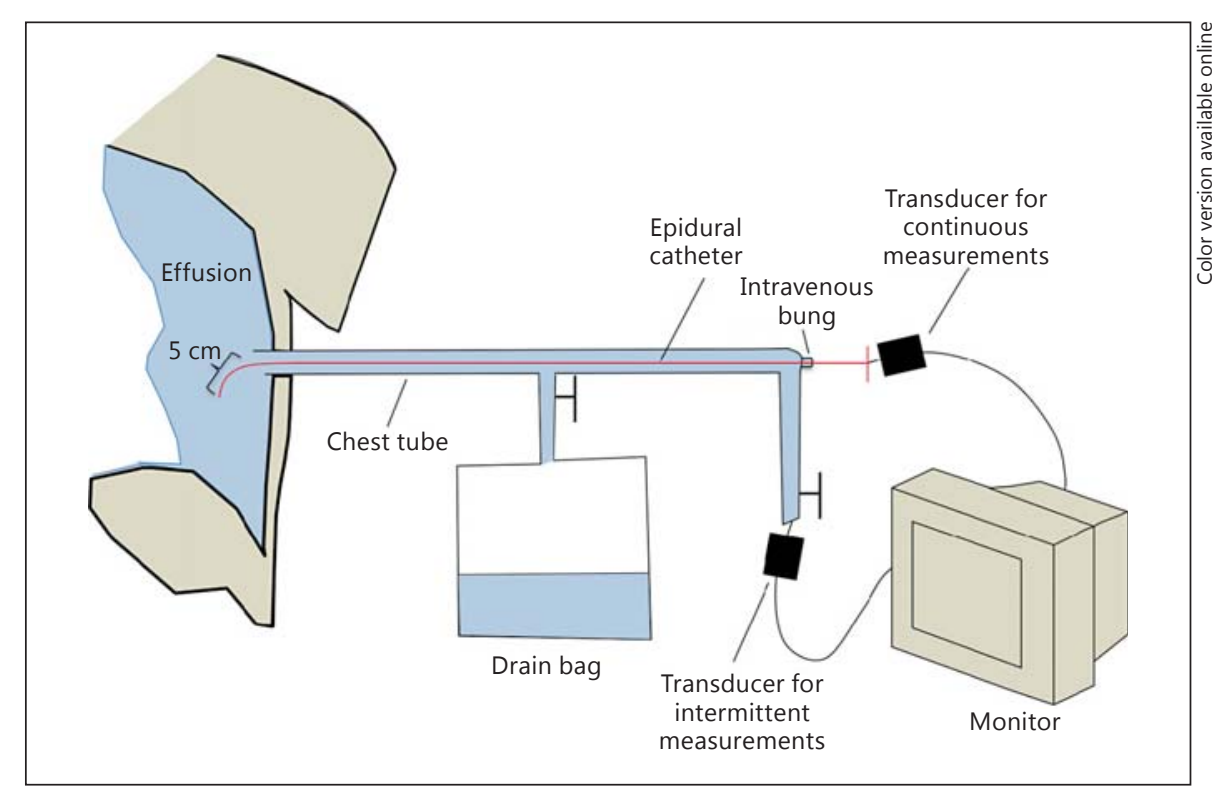

bersome and increase the complexity of the procedure. We have developed a simplified method for pleural manometry that allows for continuous recording (i.e. the continuous method) by transducing the pleural pressure via a sterile epidural catheter that is passed through the drainage tube to sit within the pleural effusion during drainage. This setup is analogous to that used for urodynamics, which has been well documented [3]. This study aims to validate this new continuous method for pleural manometry by demonstrating that the pleural elastance (PEL) measurements calculated using each technique for any given patient are equivalent.

\section{Materials and Methods}

\section{Study Design}

This was a prospective cohort study conducted at a tertiary referral hospital between May 2012 and May 2013. The study was approved by the institutional ethics committee. Consenting patients undergoing pleural drainage of at least $500 \mathrm{ml}$ were recruited.

\section{Methods}

During effusion drainage, pleural manometry was performed simultaneously with both the traditional intermittent method and the new continuous technique (fig. 1). The epidural catheter was passed through a self-sealing intravenous bung (Icon Medical, Auburn, N.S.W., Australia) at the site of entry into the chest tube, ensuring that the drainage system remained watertight. Both transducers were zeroed at the height of catheter entry through the skin. Following zeroing, the system was opened to allow the opening pleural pressure obtained from each technique to be recorded. Then, after drainage of each $100 \mathrm{ml}$ while there was still flow through the chest tube, the pressure was recorded using the continuous method. Immediately following each such recording, the flow through the tube was halted by way of a 3-way tap, while the pressure transduced using the intermittent method was noted. The system was then opened to allow a further $100 \mathrm{ml}$ drainage, and so on, until the end of the procedure. The people recording the pressures for each method were blinded to the results of the other method. Pressure-volume relationships derived from the data were inspected visually for linearity. There were no cases of a bimodal pressure-volume relationship [1] and so the $\mathrm{PEL}$, which equals the change in pressure per unit volume, was calculated using linear regression of all the data points from each patient.

Following acquisition of data using the two methods simultaneously, the feasibility of a fully automated system made possible by the new continuous recording method was demonstrated in a single patient by connecting the system to a standard urodynamics machine (Neomedix System Aquidata, Belrose, N.S.W., Australia). The epidural catheter, which was inserted through the chest tube such that its tip was within the pleural space, was connected to the machine's pressure transducer and the drainage bag was attached to a weighted cell, which transduces a change in weight (a surrogate measure of drain volume). Following zeroing of the machine, the chest tube was opened allowing free flow of pleural fluid into the drainage bag, while the changing pleural pressure was monitored via the epidural line. The setup for this procedure is shown in figure 2.

\section{Analysis}

PEL lines were calculated for each technique using standard linear regression models. These were then compared between techniques using analysis of covariance. The technique (intermittent/continuous) and the volume were entered as main effects, as well as the technique-by-volume interaction. Opening pressures and confidence intervals (CIs) for the PEL, derived with each tech- 
Fig. 2. Setup for chest drainage with pleural manometry using an automated urodynamics machine. In the insert, the epidural catheter is seen deployed inside the chest drain into the pleural space.

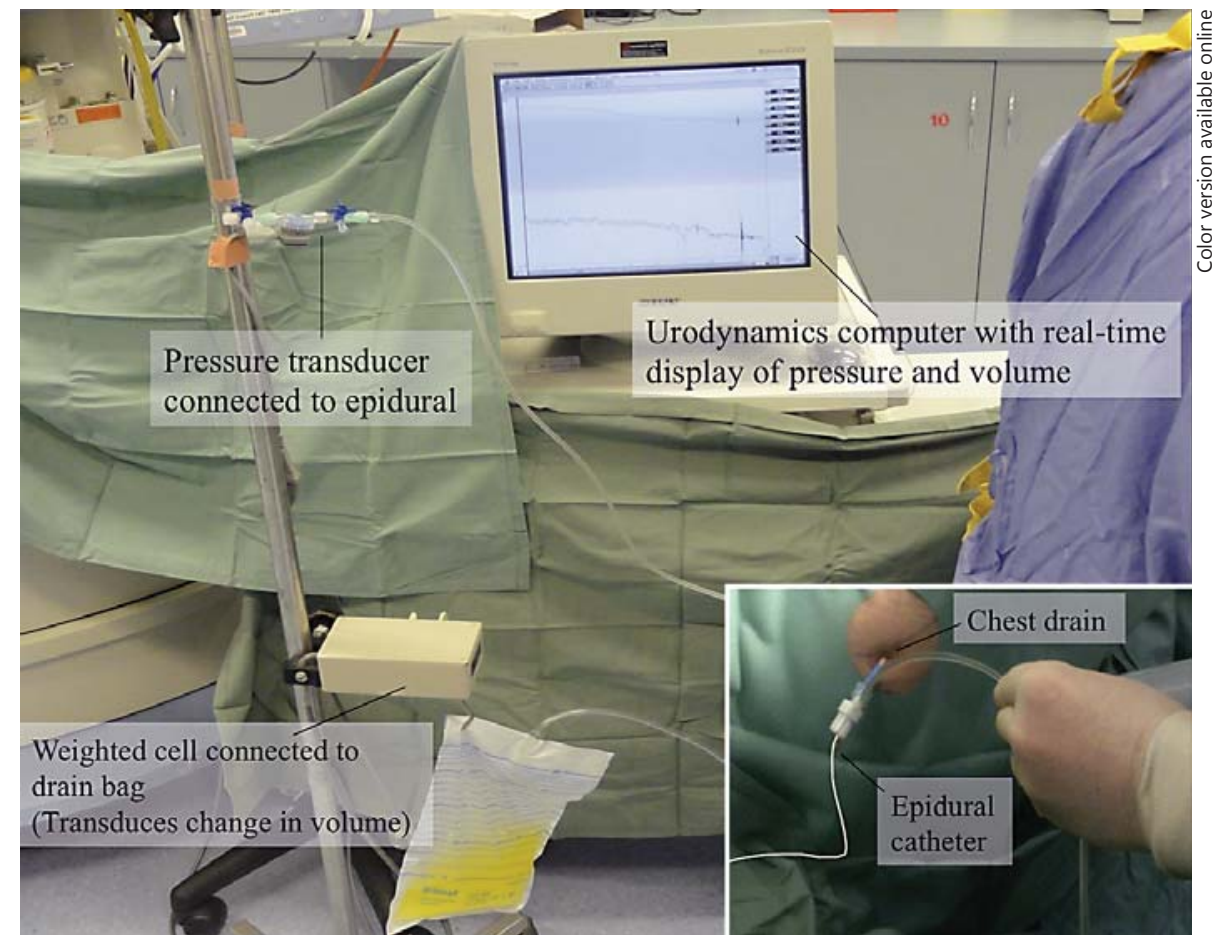

nique, were compared using Student's paired t test. Statistical analysis was performed with GraphPad Prism software version 6 (GraphPad Prism La Jolla, Calif., USA) and STATA software version 12 (StataCorp, College Station, Tex., USA).

To calculate the power of rejecting the null hypothesis that the PEL values differ between techniques for each individual, we assumed there would be 11 observations on each individual measured from a volume of $0-1$ liters in increments of 0.1 liters. Furthermore, it was clinically important that the standard deviation of the regression errors would be 1 and that there would be a difference in PEL values of $>4$. A value of 4 was chosen, as this would be the difference in PEL required to alter its classification from a normal to a trapped physiology $[2,9]$. If we sampled 10 individuals, with $\alpha=0.05$, we would have $79 \%$ power to detect at least one significant difference in PEL values between techniques.

\section{Results}

Eleven patients were recruited to the study. The median age was 70 years (range 55-73) and the median volume drained was $1,050 \mathrm{ml}$ (range 500-1,500). Seven patients had a diagnosis of pleural malignancy, 1 had pleural infection, 2 had heart failure and 1 had chronic nonspecific pleuritis. No patients had trapped lung, defined as complete separation of the visceral from the parietal pleura on postdrainage imaging.

A Method for Continuous Pleural Manometry
Table 1. Drainage parameters and comparison of PEL values derived for each patient using each technique

\begin{tabular}{llllrl}
$\begin{array}{l}\text { Patient } \\
\text { No. }\end{array}$ & Tube size, & Volume, & \multicolumn{2}{l}{ PEL $( \pm 95 \% \mathrm{CI})$} & $\mathrm{p}$ \\
\cline { 4 - 5 } & & & intermittent & continuous & \\
\hline 1 & 8 & 1.0 & $-17.6(4.0)$ & $-16.9(4.5)$ & 0.59 \\
2 & 8 & 1.5 & $-1.8(0.6)$ & $-2.3(0.8)$ & 0.30 \\
3 & 8 & 1.4 & $-8.3(1.5)$ & $-6.8(3.4)$ & 0.41 \\
4 & 8 & 1.5 & $-15.5(9.1)$ & $-15.0(8.6)$ & 0.93 \\
5 & 8 & 0.9 & $-11.1(2.9)$ & $-8.9(2.1)$ & 0.10 \\
6 & 8 & 0.9 & $-9.9(4.0)$ & $-6.8(2.2)$ & 0.12 \\
7 & 8 & 1.1 & $-9.5(5.4)$ & $-7.5(3.3)$ & 0.47 \\
8 & 8 & 1.1 & $-10.4(5.5)$ & $-11.3(4.4)$ & 0.76 \\
9 & 16 & 0.9 & $-7.5(11.7)$ & $-9.9(7.6)$ & 0.65 \\
10 & 10 & 0.5 & $-12.1(8.0)$ & $-8.6(4.7)$ & 0.42 \\
\hline
\end{tabular}

Clinical characteristics for each patient, including PEL values for each technique, are shown in table 1. Both PEL values and opening pressure were well approximated by a normal distribution. The PEL values were not significantly different between techniques for any patient, with a smallest $p$ value of 0.10 . This is reflected in the high concordance between PEL obtained from each technique, with a mean difference $(95 \% \mathrm{CI})$ of $0.98 \mathrm{~cm} \mathrm{H}_{2} \mathrm{O} / 1(-0.35$ to 2.31). An example of this is displayed in figure $3 \mathrm{a}$, 


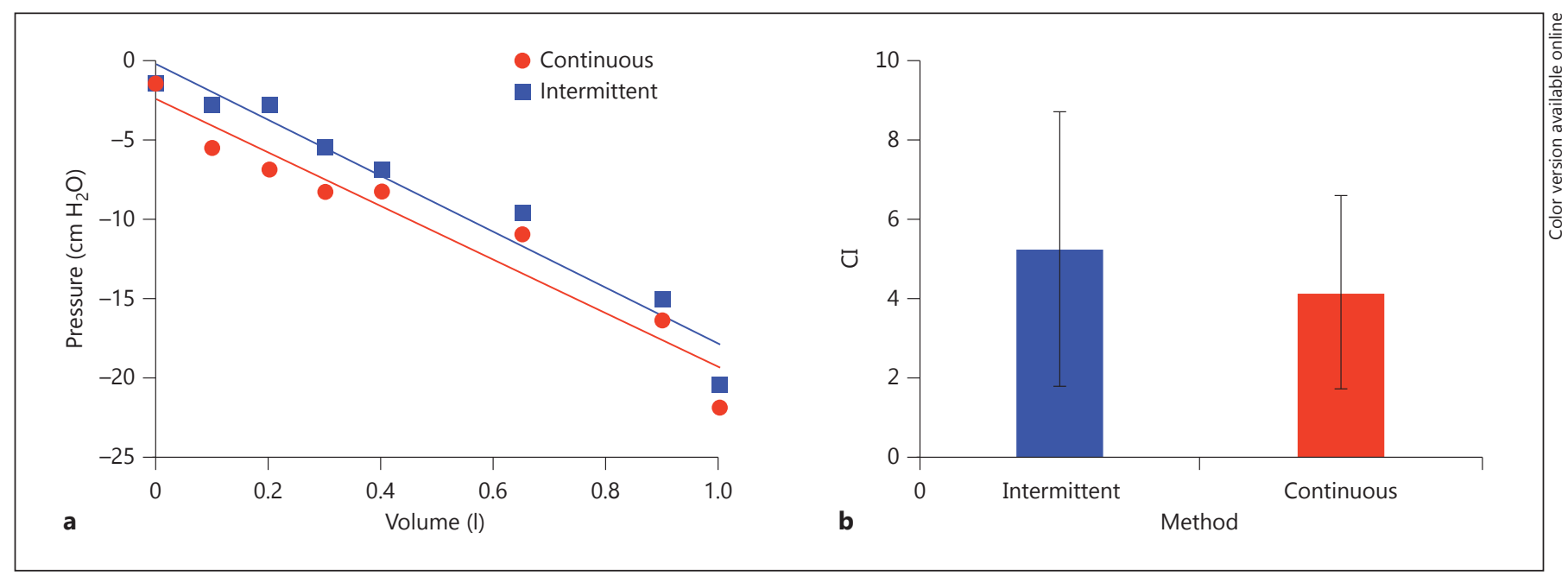

Fig. 3. a Example of pressure recordings obtained from simultaneous measurements using the two techniques with linear regression lines. b Mean CIs for the continuous compared with the intermittent technique (error bars represent standard deviation).

Table 2. Comparison of opening pressures derived from each method for each patient

\begin{tabular}{cccc}
\hline Patient No. & \multicolumn{2}{l}{ Opening pressure, $\mathrm{cm} \mathrm{H}_{2} \mathrm{O}$} & $\begin{array}{l}\text { Difference, } \\
\mathrm{cm} \mathrm{H}_{2} \mathrm{O}\end{array}$ \\
\cline { 2 - 3 } & intermittent $^{\mathrm{a}}$ & continuous $^{\mathrm{b}}$ & \\
\hline 1 & -1.4 & -1.4 & 0.0 \\
2 & 0.0 & 0.0 & 0.0 \\
3 & 1.4 & 1.4 & 0.0 \\
4 & 0.0 & 2.7 & -2.7 \\
5 & -1.4 & 4.1 & -5.5 \\
6 & 0.0 & -1.4 & 1.4 \\
7 & -1.4 & -4.1 & 2.7 \\
8 & 5.4 & 0.0 & 5.4 \\
9 & 0.0 & 0.0 & 0.0 \\
10 & 1.4 & -4.1 & 5.5 \\
\hline
\end{tabular}

${ }^{a}$ Pressure derived using the intermittent technique.

${ }^{b}$ Pressure derived using the continuous technique.

where data for patient No. 4 are displayed (p value for difference in slopes $=0.93$ ). There was a trend for PEL values derived with the continuous method to have a smaller $95 \%$ CI ( $p=0.08$; fig. $3 b)$, i.e. the PEL values obtained with the continuous method were more tightly grouped around the PEL line. There was no significant difference in opening pressures $(\mathrm{p}=0.49$; table 2$)$.

The automated real-time pressure, volume and PEL plots obtained when the system was connected to an urodynamics machine are shown in figure 4 .

\section{Discussion}

This study has documented a new method for performing pleural manometry, which allows for the continuous measurement of pleural pressure during pleural effusion drainage. The technique is simple to perform and it may make pleural manometry more accessible to the pulmonologist, increasing its use around the world.

Currently, most pulmonologists do not perform pleural manometry during pleural effusion drainage, despite its potential utility to guide safe large-volume thoracentesis and aid in the diagnosis of trapped lung. There is no commercially available equipment designed specifically for the procedure [5], and we found the traditional intermittent method of pleural manometry to be cumbersome when we were first learning the technique. Because pressure is transduced from the drainage device on the outside of the chest, flow must be halted temporarily at the time of pressure recordings. Typically, this is done every $250 \mathrm{ml}$ up to 1 liter, and then every $100 \mathrm{ml}$ thereafter [8]. Furthermore, pressures and volumes must be manually recorded and the PEL calculated at a later time. When using this traditional intermittent method, we also experienced difficulties due to air bubbles that rose from the drainage bag or underwater seal. If these migrate to sit within the drainage catheter between the point of pressure transduction and the pleural cavity, they would invalidate the pressure measurements. With the new refined method for continuous pleural manometry, because the pressure is transduced from an epidural catheter 


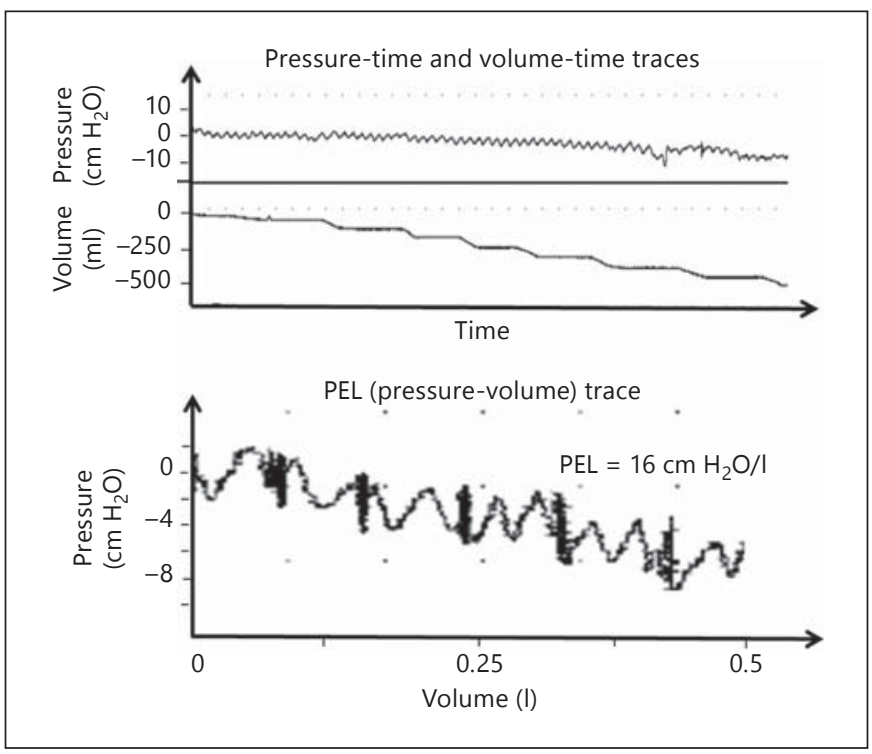

Fig. 4. Real-time pressure, volume and PEL plots obtained with a urodynamics machine.

which sits within the thoracic cavity away from pleural fluid flow, it can be recorded continuously throughout drainage and any air bubbles within the drainage device do not affect the measurements.

There was no statistically significant difference between the opening pressures measured by each technique, although this could differ by a small amount for each individual patient. This would reflect the small difference in relative height between the drainage catheter and the end of the epidural cannula, which protruded $5 \mathrm{~cm}$ beyond the end of the chest tube within the pleural space. The important point is that a small change in opening pressure would not affect the calculated PEL, as the PEL only reflects the change in pleural pressure per unit volume of fluid removed from the chest. There was also a trend towards narrower CIs for the PEL derived with the continuous technique, which probably reflects a reduction in noise that could be introduced during the repetitive opening and closing of drainage with the intermittent technique. In this study, we showed that, using the new continuous method, pleural manometry can be fully automated. This is quite simple and can be achieved by connecting the pressure transducer and drainage-volume transducer (in this case, from a weighted cell which transduces the change in weight of the drainage bag) to a computerized system that can plot pressure-volume values in real time and calculate the PEL. We used a urodynamics machine for this because it was easily available in our cen- ter, but it would not be hard to simply use a laptop computer with the appropriate software installed [10]. A further simplification could be achieved if double lumen chest tubes and thoracentesis kits were commercially available. The pressure transducer could be connected directly to the second lumen, obviating the need to insert an epidural catheter into the pleural space. At this time, however, we are only aware of a 24-Fr double lumen chest tube (Thal-Quick Double Lumen Chest Tube, Cook Medical, Bloomington, Ind., USA), which is much larger than what is required for most cases of effusion drainage.

One of the other benefits of pleural manometry is that it leads to a better understanding of the pathophysiology of pleural effusions [11] and may provide additional information on the mechanism of pneumothorax generation during effusion drainage in the presence of trapped lung. Towards the terminal part of thoracentesis in the presence of trapped lung, once the pleural pressure drops to a particular excessively negative value, the pressure is seen to partially normalize and continues to do so during further drainage, such that there is no further reduction in pleural pressure [12]. This has been hypothesized to be due to transient pressure-dependent parenchymal-pleural fistulae, but the exact mechanism could not be further investigated due to the intermittent nature of pressure recording [12] so the behavior of the pleural pressure during times of active fluid drainage could not be seen. The new method of continuous manometry overcomes this problem and may allow the pathophysiology of pneumothorax generation during effusion drainage in the presence of trapped lung to be better characterized.

There were two possible limitations to this study. Firstly, in order for a reliable pressure to be transduced from the epidural catheter, there must be no significant flow of fluid past the tip of the catheter within the pleural cavity. The catheters in this study were of a small bore, but if a larger bore was used (e.g. 16-20 Fr) and flow rates were higher, one could argue that measurements might be less reliable. However, in 1 patient in our study, a 16-Fr tube was used, and there was no significant difference between the pressure values obtained with the intermittent or the continuous method. Furthermore, in cases where a smaller caliber tube was used, a standard aspirating syringe was used to facilitate fluid drainage, which temporarily increased the negative pressure applied to the drainage catheters. Despite this, in no cases was there a significant difference between the intermittent and continuous methods. The second limitation of the study is that there were no cases of a bimodal PEL, such as can occur in the presence of trapped lung [2]. It is important to bear this 
limitation in mind, but we do not believe it would affect the performance of the continuous technique. A bimodal PEL, while usually associated with a greater pressure drop, would not be associated with an increased flow rate of fluid from the chest, which, for the reasons described above, would be needed to affect the pressure measurements. The aim of this study was not to assess the utility of the continuous method to identify the pathology associated with an abnormal or bimodal PEL, such as trapped lung, but rather to show that it gives the same results as the traditional intermittent method.

In conclusion, we have developed a new method for performing pleural manometry, which allows for the continuous measurement of pleural pressure during effusion drainage. The technique offers a simplification on the current method, and would also allow a more complete investigation of the pathophysiology of pneumothorax generation following thoracentesis in the presence of trapped lung. The technique allows for the automated recording of pleural pressures and calculation of the PEL, and would become even more straightforward if a larger range of double lumen chest tubes and thoracentesis kits became commercially available.

\section{Acknowledgements}

The primary author acknowledges support in the form of research scholarships from the University of Queensland and The Royal Brisbane and Women's Hospital Foundation.

\section{References}

$>1$ Huggins JT, Doelken P: Pleural manometry. Clin Chest Med 2006;27:229-240.

$>2$ Huggins JT, Doelken P, Sahn SA: The unexpandable lung. F1000 Med Rep 2010;2:77.

$>3$ Boshuizen RC, Sinaasappel M, Vincent AD, Goldfinger V, Farag S, van den Heuvel MM: Pleural pressure swing and lung expansion after malignant pleural effusion drainage: The benefits of high-temporal resolution pleural manometry. J Bronchology Interv Pulmonol 2013;20:200-205.

4 Feller-Kopman D, Berkowitz D, Boiselle P, Ernst A: Large-volume thoracentesis and the risk of reexpansion pulmonary edema. Ann Thorac Surg 2007;84:1656-1661.
5 Havelock T, Teoh R, Laws D, Gleeson F: Pleural procedures and thoracic ultrasound: British Thoracic Society Pleural Disease Guideline 2010. Thorax 2010;65(suppl 2):ii61-ii76.

$\checkmark 6$ Maldonado F, Mullon JJ: Counterpoint: should pleural manometry be performed routinely during thoracentesis? No. Chest 2012; 141:846-848.

7 Doelken P, Huggins JT, Pastis NJ, Sahn SA: Pleural manometry: technique and clinical implications. Chest 2004;126:1764-1769.

$>8$ Feller-Kopman D: Therapeutic thoracentesis: the role of ultrasound and pleural manometry. Curr Opin Pulm Med 2007;13:312-318.

$\checkmark$ Lan RS, Lo SK, Chuang ML, Yang CT, Tsao TC, Lee CH: Elastance of the pleural space: a predictor for the outcome of pleurodesis in patients with malignant pleural effusion. Ann Intern Med 1997;126:768-774.
0 Krenke R, Guc M, Grabczak EM, Michnikowski M, Palko KJ, Chazan R, Golczewski $\mathrm{T}$ : Development of an electronic manometer for intrapleural pressure monitoring. Respiration 2011;82:377-385.

11 Feller-Kopman D, Parker MJ, Schwartzstein RM: Assessment of pleural pressure in the evaluation of pleural effusions. Chest 2009; 135:201-209.

12 Heidecker J, Huggins JT, Sahn SA, Doelken P: Pathophysiology of pneumothorax following ultrasound-guided thoracentesis. Chest 2006; 130:1173-1184. 